\title{
p16 gene alterations in locally advanced squamous cell carcinoma of the head and neck
}

\author{
DIAN WANG ${ }^{1,3}$, JOHN C. GRECULA ${ }^{1}$, REINHARD A. GAHBAUER ${ }^{1}$, DAVID E. SCHULLER ${ }^{2}$, \\ KRIS R. JATANA ${ }^{2}$, JOHN D. BIANCAMANO ${ }^{2}$ and JAMES C. LANG ${ }^{2}$ \\ ${ }^{1}$ Division of Radiation Oncology and ${ }^{2}$ Department of Otolaryngology, Arthur G. James Cancer Hospital and \\ Richard J. Solove Research Institute, the Ohio State University Comprehensive Cancer Center, Columbus, OH, USA
}

Received September 9, 2005; Accepted November 17, 2005

\begin{abstract}
We previously documented the presence of mutations/deletions in the tumor suppressor gene $p 16$ in squamous cell carcinoma of the head and neck (SCCHN). However, the association of these p16 alterations with clinical outcome is unknown. In this study, RNA was isolated from 19 frozen SCCHN from 19 patients who were previously enrolled in the OSU intensification regimen 2. Quantitative real-time RT-PCR and direct sequencing analysis was then performed on the specimens to detect p16 gene alterations. Clinical outcome for each patient was updated and correlated with the $p 16$ alterations found. Five tumor specimens were found to have no or very low expression of p16 when compared with normal tissue. The remaining 14 tumor samples demonstrated overexpression of $p 16$ relative to the level of expression in normal tissue. Sequence analysis of the p16 RT-PCR product from these specimens allowed identification of mutational changes in the coding sequence of p16 in four of the SCCHN specimens. Subsequent analysis of clinical outcome associated with locoregional/distant failure demonstrated no correlation with either altered expression of $p 16$ or mutational status of $p 16$. Results from this study indicate that $p 16$ alterations are frequently found in this cohort of SCCHN. However, p16 alterations alone do not appear to be associated with clinical outcome.
\end{abstract}

\section{Introduction}

Realization that the underlying basis for differences in radiosensitivity of different individuals is most likely genetically determined has led to search for genetic aberrations that could be used as predictive assays. The roles of

Correspondence to: Dr Dian Wang, ${ }^{3}$ Present address: Department of Radiation Oncology, Medical College of Wisconsin, 8701 Watertown Plank Rd, Milwaukee, WI 53226, USA

E-mail: dwang@radonc.mcw.edu

Key words: squamous cell carcinoma of the head and neck, p16 gene, radiotherapy, chemotherapy oncogenes and tumor suppressor genes have been intensively studied in the development of head and neck malignancies. The tumor suppressor gene $p 16 / M T S 1 / C D K N 2$ is one of the most frequently altered genes in cancer cell lines and primary tumors including squamous cell carcinoma of the head and neck (SCCHN) (1-3). Our laboratory has also previously documented alterations to the p16 gene in SCCHN $(4,19)$. Homozygous deletion of $p 16$ has been shown to correlate with adverse prognostic features in acute lymphoblastic leukemia (5) and partial or complete loss of p16 expression correlates with the invasive stage of tumor progression in melanocytic lesions (6). However, studies that examine the correlation between p16 gene alterations and clinical outcome of patients treated for advanced head and neck carcinomas in a controlled clinical setting are lacking.

Postoperative radiotherapy (RT) is a standard option after surgical resection of locally advanced head and neck cancers. The addition of neoadjuvant and adjuvant chemotherapy has not produced any survival benefits (7-11). However, concurrent chemotherapy and radiotherapy have demonstrated some improvement in local-regional control and in overall survival in some randomized trials (12-14). Our prospective, nonrandomized controlled trial results show excellent locoregional control. Twenty-three of 25 patients (92\%) who completed the postoperative concurrent chemoradiotherapy were relapse-free at the median time of 25 months but unacceptable systemic toxicity was encountered utilizing postoperative cisplatin $\left(100 \mathrm{mg} / \mathrm{m}^{2}\right.$, days 25 and 46) and paclitaxel (135 mg/m² per $24 \mathrm{~h}$, days 24,45 and 66) (15).

In this study we report $p 16$ gene alterations in locally advanced SCCHN from 19 patients who were enrolled into the prior clinical trial. The clinical outcome for these patients is also updated.

\section{Patients and methods}

Clinical data. Patients $(n=43)$ originated from the Department of Otolaryngology, The Ohio State University, Columbus, $\mathrm{OH}$, and were enrolled into the OSU intensification protocol \#OSU9601. They all had resectable, previously untreated stage III or IV squamous cell carcinoma of the oral cavity, oropharynx, or hypopharynx or stage II squamous cell carcinomas of the hypopharynx. This study was previously 
reported (15). Briefly, it was a prospective, nonrandomized, controlled (phase II) clinical trial. Days 1-4, perioperative, slightly accelerated, hyperfractionated radiotherapy (9.1 Gy/ 7 bid fractions) to involved head and neck fields was delivered with $6 \mathrm{MV}$ photons; days $1-3$, cisplatin, $30 \mathrm{mg} / \mathrm{m}^{2}$ per day was given; day 4, patients had surgical resection and intraoperative electron boost $(7.5 \mathrm{~Gy})$ to the area of closest surgical margin; days $45-52$, postoperative radiotherapy (40 Gy/20 fractions/ $6 \mathrm{MV}$ to the primary site and bilateral upper neck regions and $45 \mathrm{~Gy} / 20$ fractions/6 MV to the supraclavicular areas); days 24,45 , and 66 , paclitaxel, $135 \mathrm{mg} / \mathrm{m}^{2}$ over $24 \mathrm{~h}$ was delivered with routine granulocyte colony-stimulating factor support; days 25 and 46, cisplatin, $100 \mathrm{mg} / \mathrm{m}^{2}$ was delivered.

Twenty-five of 43 patients completed intensification regimen 2. Thirty-six patients completed the planned radiotherapy but missed one or two cycles of postoperative chemotherapy due to increased toxicity of our systemic regimen. Thirty-three of 36 patients provided fresh tissue for our molecular study. The clinical outcome for these 33 patients has been recently updated at a median follow-up time of 41 months (3 locoregional failure, 2 locoregional/distant recurrence and 4 distant failure). In this study, we chose a subset of 19 patients including the majority of locoregional recurrences and distant failures (Table I).

$R N A$ extraction and quantitative real-time $R T-P C R$. Tissue samples were provided by the Cooperative Human Tissue Network funded by the National Cancer Institute. Total RNA was extracted from tumor samples from 19 patients as previously described (4). All patient specimens were subject to quantitative Real-Time RT-PCR in order to accurately quantify the level of expression of p16. Quantitative RealTime RT-PCR was performed using the $\Delta \Delta_{\text {CT }}$ (Applied Biosystems, Foster City, CA) comparative method utilizing HPRT as the normalization control gene. The expression level of p16 in all tumor specimens was then compared with the expression level obtained from a human normal RNA pool obtained by pooling equivalent amounts of RNA obtained from 10 normal specimens of upper-aerodigestive tract epithelium. Reverse transcription was performed using Multiscribe Reverse Transcriptase (Applied Biosystems) utilizing $100 \mathrm{ng}$ of total RNA. Real-Time PCR amplification was subsequently performed using the TaqMan PCR Kit (Applied Biosystems). Primers used for PCR amplification and fluorescent probes were designed utilizing Primer Express software (Applied Biosystems), p16 upper: 5'TGCCCAACGCACCGA-3', lower: 5'-GGGCGCTGCCCA TCA-3' and probe: 5'-TAGTTACGGTCGGAGGCCGAT CCA-3', HPRT upper: 5'-CGGCTCCGTTATGGCG-3', lower: 5'-GGTCATAACCTGGTTCATCATCAC-3' and probe: 5'-CGCAGCCCTGGCGTCGTGA-3'. All RNA samples were subject to DNase treatment prior to PCR amplification; however, primers were additionally selected to produce an intron-spanning PCR product, precluding amplification of contaminating DNA. Real-Time PCR amplification was performed in a Perkin-Elmer/Applied Biosystems 5700 machine. Method for study comprised use of fluorogenic 5' nuclease chemistry, utilizing an oligonucleotide probe 5' labeled with fluorochrome 6-carboxy- fluorescein (6-FAM) and a quencher fluorochrome, 6carboxy-tetramethyl-rhodamine (TAMRA).

\section{Results}

Clinical treatment outcome. All 19 patients had resectable, previously untreated stage III or IV squamous cell carcinoma of the oral cavity, oropharynx, or hypopharynx or stage II squamous cell carcinomas of the hypopharynx. Location and staging information are summarized in detail in Table I. These patients have a median age of 56 years (range of 3269 years) with median follow-up time of 46 months. Fifteen patients were treated with planned multimodality therapies per protocol. Four patients received the scheduled preoperative CDDP and completed the planned radiotherapy but missed one or two cycles of postoperative CDDP and/or paclitaxel. All patients had a median radiotherapy break of 1 day with a range of 0 to 13 days. Four patients presented with local-regional failure within two years and 4 with distant failure (liver and lungs) (Table I). One patient was found to have locoregional failure and distant lung metastases at the same time. All locoregional failures were within the radiotherapy treatment fields. Considering the clinical presentations, short time to progression, CT scans and histology, these 4 locoregional recurrences were considered infield tumor relapses although the possibility of a second primary carcinoma can not be completely excluded.

Alterations to p16 in locally advanced SCCHN of the head and neck. RNA was extracted from 19 SCCHN specimens. Semi-quantitative RT-PCR analysis demonstrated undetectable, or very low, expression of $p 16$ in 5/19 SCCHN specimens (Table I). The mechanisms responsible for downregulation of the 16 gene have been well documented and comprise gene deletion or hypermethylation $(1-3,17,18)$. Lack of expression in these 5 tumor specimens may be due to either mechanism, however, lack of available DNA precludes further study, and these tumor samples were not subject to subsequent sequencing analysis. The remaining 14 specimens did express measurable levels of $p 16$ RNA, as detected by semi-quantitative RT-PCR, and the RT-PCR amplification products were then subject to sequence analysis. The sequence analysis has been reported in detail elsewhere (19) and allowed detection of pl6 gene alterations in 4 of the tumor specimens. Two specimens, case \#333 and case \#471, comprise an identical deletion of $74 \mathrm{bp}$ in the coding region of exon 2 (TCGCAC/ACATCC), causing a translational frame-shift. These specimens were obtained from separate individuals (males, age 42 and 48 respectively) and RNA was isolated 10 months apart, precluding any cross contamination of these specimens. A third tumor sample (\#476) contained a deletion of a single nucleotide at codon 130, resulting in a p16 translational frame-shift. A fourth specimen possessed a silent mutation in codon 70 (CCCAAC to CCTAAC) of the p16 gene. However, as described elsewhere (19), this change results in the generation of a premature stop codon in the reading frame of the overlapping $\mathrm{p} 14^{\mathrm{ARF}}$ gene and has been shown to be germline in origin (19). No additional p16 gene alterations were found in the remaining 10 tumor samples shown to express $p 16$. Previous studies from our laboratory have described semi-quantitative analysis of $p 16$ 
Table I. Clinical data and $p 16$ status of 19 patients.

\begin{tabular}{|c|c|c|c|c|c|c|c|c|c|c|}
\hline Case & $\mathrm{A} / \mathrm{S} / \mathrm{R}$ & Primary site & $\begin{array}{l}\text { AJCC Patho- } \\
\text { logy stage } 5\end{array}$ & $\begin{array}{l}\text { Living } \\
\text { status }\end{array}$ & $\begin{array}{l}\mathrm{F} / \mathrm{U} \\
(\mathrm{mo.})\end{array}$ & $\begin{array}{c}\mathrm{LR} \\
(\mathrm{mo} .)\end{array}$ & $\begin{array}{c}\mathrm{DF} \\
(\mathrm{mo} .)\end{array}$ & $\begin{array}{l}\text { Mutation/ } \\
\text { deletion }^{\mathrm{a}}\end{array}$ & $\begin{array}{c}\text { mRNA } \\
\text { expression }^{\mathrm{a}}\end{array}$ & $\begin{array}{l}\text { Postop- } \\
\text { chemo }\end{array}$ \\
\hline
\end{tabular}

\begin{tabular}{|c|c|c|c|c|c|c|c|c|c|c|}
\hline 284 & $60 / \mathrm{m} / \mathrm{w}$ & Tonsilar fossae & IV-T3N2cM0 & DWD & 14 & 12 & & & Elevated & 3 CDDP $\& 2$ Tax \\
\hline 316 & $63 / \mathrm{m} / \mathrm{W}$ & Pyriform sinus & IV-T2N2bM0 & dwoD & 9 & & & & Elevated & 3 CDDP \& 3 Tax \\
\hline 320 & $42 / \mathrm{m} / \mathrm{w}$ & Tonsil & IV-T2N2AM0 & AWoD & 56 & & & & Elevated & 3 CDDP $\& 3$ Tax \\
\hline 319 & 60/F/W & Pyriform sinus & IV-T2N2BM0 & AWD & 55 & & $43 \mathrm{mo}$ & & Elevated & 3 CDDP $\& 3$ Tax \\
\hline 326 & $64 / \mathrm{M} / \mathrm{W}$ & Hypopharynx & IV-T3N2CM0 & dwoD & 46 & & & & Elevated & 3 CDDP $\& 3$ Tax \\
\hline 333 & $42 / \mathrm{M} / \mathrm{W}$ & $\begin{array}{l}\text { Mandibular } \\
\text { gingiva }\end{array}$ & IV-T4N3M0 & DWD & 7 & 6 & & Deletion & Elevated & 1CDDP\&1 Tax \\
\hline 334 & 60/F/W & Soft palate & III-T3N1M0 & dwoD & 44 & & & & Elevated & 3 CDDP \&3 Tax \\
\hline 338 & $50 / \mathrm{M} / \mathrm{B}$ & Tongue & IV-T4N1M0 & AWoD & 60 & & & & $\mathrm{AOL}$ & 3 CDDP $\& 3$ Tax \\
\hline 339 & $47 / F / W$ & $\begin{array}{l}\text { Mandibular } \\
\text { gingiva }\end{array}$ & II-T2N0M0 & AWoD & 57 & & & & $\mathrm{AOL}$ & 3 CDDP\&3 Tax \\
\hline 396 & $65 / \mathrm{M} / \mathrm{W}$ & Tonsil & IV-T2N2bM0 & DWD & 22 & 22 & & & AOL & 3 CDDP $\& 3$ Tax \\
\hline 343 & $64 / \mathrm{M} / \mathrm{W}$ & Pyriform sinus & III-T2N1M0 & AWoD & 53 & & & & $\mathrm{AOL}$ & 3 CDDP \& 1 Tax \\
\hline 344 & 69/F/B & Base tongue & IV-T2N2BM0 & AWoD & 48 & & & & Elevated & 3 CDDP $\& 3$ Tax \\
\hline 354 & $55 / \mathrm{M} / \mathrm{W}$ & Base tongue & IV-T2N2AM0 & DWD & 22 & 33 & & & Elevated & 3 CDDP \&3 Tax \\
\hline 371 & $69 / \mathrm{F} / \mathrm{B}$ & Soft palate & IV-T3N2BM0 & DWD & 23 & 23 & $23 \mathrm{mo}$ & & Elevated & 3 CDDP $\& 3$ Tax \\
\hline 372 & $55 / \mathrm{F} / \mathrm{W}$ & Base tongue & IV-T4N2BM0 & AWoD & 56 & & & & Elevated & 3 CDDP $\& 3$ Tax \\
\hline 377 & $56 / \mathrm{M} / \mathrm{W}$ & Gingiva & II-T2N0M0 & DWD & 43 & 49 & & & AOL & 3 CDDP \&3 Tax \\
\hline 403 & $47 / \mathrm{M} / \mathrm{W}$ & Base tongue & IV-T2N2BM0 & AWoD & 54 & & & Mutation & Elevated & 2 CDDP \& 1 Tax \\
\hline 471 & $49 / \mathrm{M} / \mathrm{W}$ & Pyriform sinus & III-T2N1M0 & AWoD & 56 & & & Deletion & Elevated & 3 CDDP \&3 Tax \\
\hline 476 & $32 / \mathrm{M} / \mathrm{W}$ & Tongue & III-T1N1M0 & AWoD & 48 & & & Deletion & N/A & 3 CDDP $\& 3$ Tax \\
\hline
\end{tabular}

${ }^{a}$ p16 Alteration status; A/S/R, age/sex/race; DWD, dead with disease; dwoD, dead without disease; AWoD, alive without disease; F/U, follow-up months from the day 1 therapy; LR, time of loco-regional failure from the day 1 therapy; DF, time of distant failure from the day 1 therapy; AOL, abscence or very low expression of p16; Postop-chemo, postoperative chemotherapy; CDDP, cisplatin; TAX, paclitaxal. Numbers indicate postoperative cycles of chemotherapy.

expression levels in SCCHN specimens $(4,19)$. These studies indicated that p16 levels may be elevated in some cases of SCCHN. This result is surprising since pl6 is a well-known tumor suppressor gene whose function is frequently inactivated in cancer. In order to more accurately analyze the expression levels of $p 16$ in SCCHN, and confirm that p16 overexpression is a true phenomenon in SCCHN, we performed quantitative Real-Time RT-PCR on 13 of the 14 specimens (no remaining RNA was available for specimen \#476) used for sequence analysis and on 1 specimen (\#377) that lacked p16 expression as previously measured by semi-quantitative RTPCR. The results are shown in Fig. 1. The expression level of p16 in each tumor specimen was determined and is shown on a linear scale, in each case, as a ratio to the level of expression of normal control RNA (pool of 10 pathologically normal epithelial tissues obtained from the oropharynx) after normalization to the expression level of ubiquitously expressed housekeeping gene HPRT. Using this quantitative Real-Time RT-PCR assay, as described by Livak and
Schmittgen (21), baseline expression is equivalent to the expression level found in the normal oropharyngeal control tissue. Thus all of the selected specimens with the exception of specimen \#377 express p16 RNA at a level above the expression in normal tissue (equivalent to baseline). The results confirm frequent elevation of p16 expression in tumor specimens. The level of expression of $p 16$ varies between specimens over a 10-fold range when low expressors (\#316 and \#326), are compared with the highest p16 expressing tumor (\#334). As expected, some tumors express no detectable p16 RNA as exemplified by specimen \#377. Therefore, these data confirm our earlier observations and demonstrate that overexpression of p16 is indeed a common phenomenon in SCCHN.

Correlation of p16 alterations with clinical treatment outcome. Clinical outcome of patients for whom p16 status has been determined is shown in Table I. The results show no obvious correlation between either $p 16$ mutational status or $p 16$ 


\section{p16 Expression in SCCHN}

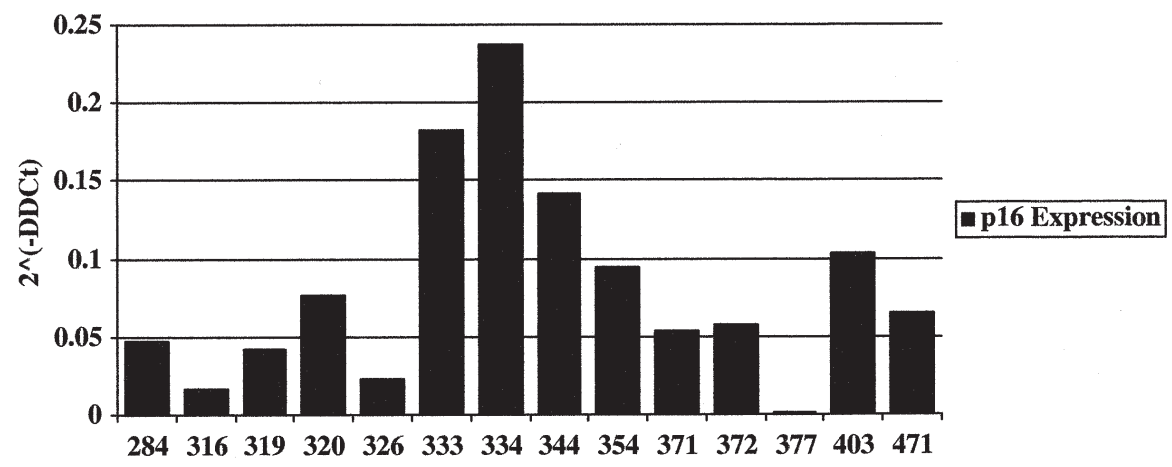

Figure 1. Quantitative Real-Time RT-PCR analysis of p16 expression in specimens of SCCHN. A representative experiment from 3 duplicate experiments is shown. The expression level of $p 16$ is shown in each case as the average value of duplicate samples, represented on a linear scale.

expression level and clinical outcome. In this study, our data demonstrate no association between locoregional recurrence and/or distant failures and age, sex, tumor location or stage.

\section{Discussion}

Our institution initiated the first intensification trial in 1993 using perioperative chemoradiotherapy, surgery and postoperative chemoradiotherapy (intensification regimen 1). This initial trial demonstrated excellent patient compliance (92\%) and excellent locoregional control (no tumor recurrence at a median time of risk of 18.6 months). Our recent update upholds the excellent locoregional control (OSU internal communication). The second trial was initiated in 1996 using intensification regimen 2. Considering the excellent local control in the intensification regimen 1 , the radiotherapy schema was not changed in the intensification regimen 2 . Due to a relatively high rate $(5 / 37)$ of distant metastasis in the initial trial, we added paclitaxel to the postoperative regimen. Also, the dosage of the perioperative cisplatin was changed, from $80 \mathrm{mg} / \mathrm{m}^{2}$ over 80 - $\mathrm{h}$ continuous infusion to $30 \mathrm{mg} / \mathrm{m}^{2}$ per day for 3 days, to allow outpatient administration. Patient compliance (39/43) and local control (23/25) were reportedly excellent at the median time (25 months) at risk (15). 25/43 patients completed scheduled concurrent chemoradiotherapy. The present study was focused on a subset of 19 of these patients, including the majority of locoregional and/or distant failures, for clinical outcome correlation. Only one locoregional failure and one distant failure were not included in this study.

Alterations to the p16 gene have been intensively studied over the past decade in a variety of malignancies including squamous cell carcinomas of the head and neck. The present study also demonstrates the presence of p16 gene alterations in a cohort group of locally advanced squamous cell carcinomas of the head and neck and is consistent with many previous studies describing the presence of frequent alterations of the pl6 gene in this disease $(1-4,19)$. In this investigation, we have additionally studied whether a correlation exists between clinical outcome and p16 status. Clinical outcome of loco- regional recurrences and/or distant failures were not found to be associated with altered (high or low) levels of expression of $p 16$. In this study we used the comparative $\Delta \Delta_{\mathrm{CT}}$ method for quantitative Real-Time RT-PCR measurements, to accurately measure p16 expression levels in tumor tissue compared to levels in a pooled normal tissue sample. Using this method we confirmed our earlier unexpected observations of frequent p16 overexpression, previously measured by the less precise method of semi-quantitative RT-PCR. Thus, the present study demonstrates that overexpression of $p 16$ is a frequently observable event in SCCHN. p16 is a tumor suppressor gene and therefore the observed overexpression of p16 in SCCHN is surprising. However, a possible explanation for this phenomenon, based on cell line studies, has previously been described (20) and suggests that overexpression of p16 occurs as a direct response to inactivation of the retinoblastoma gene and associated abrogation of the negative feedback loop responsible for retinoblastoma downregulation of p16 expression.

Sequence analysis of RT-PCR products from the SCCHN specimens has previously allowed the identification of 4 specimens harboring p16 alterations (19). In the present study we have investigated whether the mutational status of p16 in these specimens correlates with clinical outcome. Specimen \#333 (containing a deletion of $74 \mathrm{bp}$ in the coding region of exon 2) was isolated from a patient who presented with a very aggressive clinical course. Locoregional failure was observed within 4 months following the combined modality treatment. This deletion was also confirmed in a biopsied recurrent tumor sample (\#301) (19) obtained from the same patient. In contrast, tumor specimen \#471 contained a deletion identical to that of \#333, yet this patient is still alive without evidence of disease at the most recent follow-up (56 months). In addition, the patient donor of specimen \#476, containing a single nucleotide deletion (frame-shift) of $p 16$, is alive at his most recent follow-up of 48 months. Specimen \#403 harbors a point mutation in $p 16$. However, this alteration is a silent mutation and has no effect on the pl6 coding sequence. As described above, this mutation, in contrast, results in truncation of the $\mathrm{p} 14^{\mathrm{ARF}}$ polypeptide. This specimen is the only $\mathrm{p} 14^{\mathrm{ARF}}$ specific mutation we have observed in 84 cases of SCCHN so far examined (19). This mutation does not appear to be 
associated with poor prognosis since the patient is alive without disease 54 months post-surgery. Therefore, overall, alteration to $p 16$ shows no detectable association with clinical treatment outcome for locoregional recurrence and/or distant failure. Prior studies have shown either partial or complete deletion of $p 16$ correlated with adverse prognostic features (increased leukemic cell mass and white blood cell counts) (5) and that loss of p16 is more frequently found in metastatic lesions of melanocytic origin $(72 \%)$ than in primary tumors $(52 \%)$ of the same type (6). In our present study, p16 analysis was performed on 19 tumor samples from a subset of patients enrolled into our prior prospective clinical trial. All these patients have been seen for regular follow-up in our institution after completion of their multimodality therapies. Among these 19 patients, 3 patients had locoregional recurrences, 3 patients had distant metastasis and 1 patient was found to have both locoregional recurrence and distant metastasis at the same time. However, we find no correlation between $p 16$ alterations and clinical treatment outcome in this well-controlled setting. This observation may not be unexpected, and may be explained by the possibility that tumors showing no detectable change in p16, may possess alterations in the same biochemical pathway (retinoblastoma) through mutational changes or altered expression levels of other key genes that regulate this pathway, including cyclin D1, CDK4 or the retinoblastoma gene itself.

In summary, the present study has demonstrated the presence of $p 16$ alterations in locally advanced squamous cell carcinoma of the head and neck. However, in this study, we find no detectable correlation between p16 gene alterations (expression level and mutation/ deletion status) and clinical outcome.

\section{Acknowledgements}

This study was supported in part by grants P30CA16058 from the National Cancer Institute and PO1 DE12704 from the National Institute for Dental and Craniofacial Research.

\section{References}

1. Zhang S-Y, Klein-Szanto A, Sauter ER, et al: Higher frequency of alterations in the $p 16 / \mathrm{CDKN} 2$ gene in squamous cell carcinoma cell lines than in primary tumors of the head and neck. Cancer Res 55: 505-509, 1955.

2. Reed AL, Califano J, Cairns $\mathrm{P}$, et al: High frequency of $p 16$ (CDKN2/MTS-1/INK4A) inactivation in head and neck squamous cell carcinoma. Cancer Res 56: 3630-3633, 1996.

3. Olshan AF, Weissler MC, Pei H, et al: Alterations of the p16 gene in head and neck cancer: frequency and association with p53, PRAD-1 and HPV. Oncogene 14: 811-818, 1997.
4. Lang JC, Tobin EJ, Knobloch TJ, et al: Frequent mutation of p 16 in squamous cell carcinoma of the head and neck. Laryngoscope 108: 923-928, 1998.

5. Fizzotti M, Cimino G, Pisegna S, et al: Detection of homozygous deletions of the cyclin-dependent kinase 4 inhibitor ( $p 16)$ gene in acute lymphoblastic leukemia and association with adverse prognostic features. Blood 10: 26852690, 1995.

6. Reed JA, Loganzo F, Shea CR, et al: Loss of expression of the $p 16 /$ cyclin-dependent kinase inhibitor 2 tumor suppressor gene in melanocytic lesions correlates with invasive stage of tumor progression. Cancer Res 55: 2713-2718, 1995.

7. Adelstein DJ, Tan EH and Lavertu P: Treatment of head and neck cancer: the role of chemotherapy. Crit Rev Oncol Hematol 24: 97-116, 1996 .

8. Vokes EE, Weichselbaum RR, Lippman SM, et al: Head and neck cancer. N Engl J Med 328: 184-194, 1993.

9. Fu KK: Combined-modality therapy for head and neck cancer. Oncology 11: 1781-1796, 1997.

10. Laramore GE, Scott CB, Al-Sarraf M, et al: Adjuvant chemotherapy for resectable squamous cell carcinomas of the head and neck: report on intergroup study 0034. Int J Radiat Oncol Biol Phys 23: 705-713, 1992.

11. Head and Neck Contracts Program: Adjuvant chemotherapy for advanced head and neck squamous carcinoma. Final report of the head and neck contacts program. Cancer 60: 301-311, 1987.

12. Adelstein DJ, Saxton JP, Lavertu P, et al: A phase III randomized trial comparing concurrent chemotherapy and radiotherapy with radiotherapy alone in resectable stage III and IV squamous cell head and neck cancer: preliminary results. Head Neck 19: 567-575, 1997

13. Brizel DM, Albers ME, Fisher SR, et al: Hyperfractionated irradiation with or without concurrent chemotherapy for locally advanced head and neck cancer. N Engl J Med 338: 1798-1804, 1998.

14. Calais G, Alfonsi M, Bardet E, et al: Randomized trial of radiation therapy versus concomitant chemotherapy and radiation therapy for advanced stage oropharynx carcinoma. J Natl Cancer Inst 91: 2081-2086, 1999.

15. Grecula JC, Schuller DE, Rhodes CA, et al: Intensification regimen 2 for advanced head and neck squamous cell carcinomas. Arch Otolaryngol Head Neck Surg 125: 1313-1318, 1999.

16. Okamoto A, Demetrick DJ, Spillare EA, et al: P16INK4 mutations and altered expression in human tumors and cell lines. Cold Spring Harb Symp Quant Biol 59: 49-57, 1994.

17. Merlo A, Herman JG, Mao L, et al: 5' CgG island methylation is associated with transcriptional silencing of the tumor suppressor p16/CDKN2/MTS1 in human cancers. Nat Med 1: 686-692, 1995.

18. Lilischkis R, Sarcevic B, Kennedy C, et al: Cancer-associated mis-sense and deletion mutations impair plolnk4 CDK inhibitary activity. Int J Cancer 66: 249-254, 1996.

19. Lang JC, Borchers J, Danahey D, et al: Mutational status of overexpressed $p 16$ in head and neck cancer: evidence for germline mutation of p16/p14 ${ }^{\mathrm{ARF}}$. Int J Oncol 21: 401-408, 2002.

20. Parry D, Bates S, Mann DJ, et al: Lack of cyclin D-Cdk complexes in Rb-negative cells correlates with high levels of p16INK4/MTS1 tumor suppressor gene product. Eur Mol Biol Org J 14: 503-511, 1995.

21. Livak KJ and Schmittgen TD: Analysis of relative gene expression data using Real time quantitative PCR and the $2-\Delta \Delta \mathrm{CT}$ method. Methods 25: 402-408, 2001. 